\title{
EDITORIAL
}

\section{Are there sex differences in risk factors for coronary heart disease? Maternal versus paternal transmission}

\author{
R F Redberg
}

Heart 2003:89:817-818

Does maternal or paternal transmission in family history play a greater role in determining an individual's overall risk for coronary heart disease?
Correspondence to: Rita F Redberg, MD, UCSF National Center of Excellence in Women's Health, 505 Parnassus Avenue, Suite M-1180, School of Medicine, Division of Cardiology, San Francisco, California 94143-0124, USA; redberg@medicine.ucsf.edu
$\mathrm{P}$ arents are held accountable for many things in their children's lives-both good and bad. The relative importance of the mother's role versus the father's role is often debated, and the relative importance of parental transmission to heart disease risk is no exception. As with many traits, there is clearly a role of nature and nurture in the occurrence of heart disease. The role of the intrauterine environment in determining coronary heart disease (CHD) risk is not nearly as well defined as the role of traditional cardiac risk factors. It is estimated from large studies such as MRFIT, the Nurses Health Study, and Chicago Western Electric study that the major independent conventional risk factors can account for more than $80 \%$ of coronary heart disease. Premature family history (mother $<65$ years, father $<55$ years) of CHD is a risk factor for CHD. While family history is clearly not modifiable, its importance is in determining overall risk. Current American Heart Association and European Society of Cardiology guidelines support tailoring the aggressiveness of risk factor reduction to the number of risk factors present. When premature family history is present, then other modifiable risk factors such as hypertension, obesity, or hypercholesterolaemia should be treated more aggressively.

\section{ENDOGENOUS V EXOGENOUS SUPPLEMENTATION: A VARIATION ON NATURE $V$ NURTURE}

The other non-modifiable risk factors-age, race, and sex-must also be considered in terms of assessing overall CHD risk. Sex differences exist in the epidemiology of CHD. On average the onset of CHD in women is 10 years later than in men. The reasons for this difference are not well understood. The traditional thinking that this later onset in women was caused by the protective effect of female hormones was thrown into disarray by the Heart and Estrogen/progestin Replacement Study ${ }^{1}$ and the recent US Women's Health Initiative results. There is clearly still more to learn about the effects of naturally occurring and secreted oestrogen versus supplemental treatment in the mechanisms of cardiovascular protection. Our experience with the role of anti-oxidant vitamins and $\mathrm{CHD}$ risk showed striking differences in CHD protection between trials using vitamin supplementation (Heart Protection Study, CHAOS) and the reduced risks seen in people consuming diets naturally high in antioxidant vitamins (Lyon study). We await results from other ongoing studies of hormone replacement such as the Women's International Study of long-Duration Oestrogen after Menopause and the estrogen only arm of the Women's Health Initiative.

\section{UNIVERSITY OF GLASGOW FOLLOW UP STUDY}

Some cardiac risk factors, such as type II diabetes, are known to be transmitted preferentially through the mother, presumably because of intrauterine environmental factors. There is a dearth of data on the relative importance of maternal versus paternal transmission of CHD risk. In this issue of Heart, Kinra and colleagues seek to fill in that gap. ${ }^{2}$ They present 43 years of follow up data from a prospective cohort study of 8402 male students at the University of Glasgow. Data were collected when the students were between the ages of 16-30 years. While premature family history of CHD was clearly a risk factor for CHD, there was no differential transmission of CHD according to paternal or maternal history. Thus, responsibility for increased CHD risk seems to be shared equally by both parents. The large numbers and long term follow up are strengths of this study.

The lack of female students in the dataset limits the applicability of the data. In addition, a "history of angina" was sufficient to be considered a positive family history as opposed to using only hard CHD events. Finally, presumably some parents developed CHD after the survey period, which was not recorded. However, earlier history of parental myocardial infarction is a stronger risk factor than parental myocardial infarction at older ages, ${ }^{3}$ so presumably this truncation would not change overall results.

\section{RISK FACTOR REDUCTION STRATEGIES}

The results of Kinra and colleagues ${ }^{2}$ reinforce the need to carefully counsel all patients with a positive family history of coronary disease on aggressive risk factor reduction-including smoking cessation, a heart healthy diet rich in fruits and

Abbreviations: CHAOS, Cambridge heart anti-oxident study; CHD, coronary heart disease; EUROASPIRE, European action on secondary prevention through intervention to reduce events; MRFIT, multiple risk factor intervention trial 
vegetables, regular physical activity, and weight loss and pharmacotherapy for hypertension and hypercholesterolaemia when appropriate. Data from EUROASPIRE and the American Behavioral Risk Factor Surveillance System suggest we continue to have much room for improvement in these areas. For example, in EUROASPIRE, 37\% of patients had a family history of premature coronary heart disease in a first degree blood relative, but only $21 \%$ of patients reported being advised to have their relatives screened for coronary risk factors. ${ }^{4}$ This European survey has demonstrated a high prevalence of modifiable risk factors in coronary heart disease patients. While we continue to learn more about risk factors for CHD, it is most important to also work on improvement in risk factor reduction. The global obesity epidemic ${ }^{5}$ and increase in concomitant diabetes, now known as "diabesity", reminds us of the tremendous amount of work we have to do in this area. Recent data from the Centers for Disease Control and Prevention show that $60 \%$ of Americans are overweight or obese and only $20 \%$ meet recommended physical activity guidelines.
There is considerable potential for cardiologists and physicians to work with patients to further reduce coronary heart disease morbidity and mortality and improve patient's chances of survival.

\section{REFERENCES}

1 Hulley S, Grady D, Bush T, et al. Randomized trial of estrogen plus progestin for secondary prevention of coronary heart disease in postmenopausal women. Heart and estrogen/progestin replacement study (HERS) research group. JAMA 1998:280:605-13.

2 Kinra S, Davey Smith G, Okasha M, et al. Is maternal transmission of coronary heart disease risk stronger than paternal transmission? Heart 2003;89:834-8.

3 Sesso HD, Lee IM, Gaziano JM, et al. Maternal and paternal history of myocardial infarction and risk of cardiovascular disease in men and women. Circulation 2001;104:393-8.

4 European Society of Cardiology. EUROASPIRE. A European Society of Cardiology survey of secondary prevention of coronary heart disease: principal results. EUROASPIRE study group. European action on secondary prevention through intervention to reduce events. Eur Heart J 1997; 18:1569-82

5 Mydans S. Clustering in cities, Asians are becoming obese. New York Times 13 March 2003.

\section{IMAGES IN CARDIOLOGY}

Patency of multiple coronary artery bypass grafts demonstrated by multislice CT

A 73 year old man with chest pain presented to our hospital where a conventional coronary angiogram revealed severe stenosis of the right coronary artery and the obtuse marginal branch (OM) and total occlusion of the left anterior descending branch (LAD) and left circumflex branch. An emergent coronary artery bypass graft was performed in which the left internal thoracic artery (LITA) was connected to the distal portion of the LAD, and two saphenous vein grafts (SVG) were connected to the posterior lateral branch (PL) and OM from the aortic root.

To evaluate the patency of the bypass grafts, ECG gated multislice computed tomography (CT) (Light Speed Ultra, General Electric, Milwaukee, Wisconsin, USA) was performed with a $1.25 \mathrm{~mm}$ slice thickness, helical pitch 3.25. Follow- ing intravenous injection of $100 \mathrm{ml}$ of iodinated contrast material $(350 \mathrm{mgI} /$ $\mathrm{ml})$, CT scanning was performed with retrospective ECG gated reconstruction. After acquisition, volume data were extracted and three dimensional volume rendering images were generated (M900 Zio Tokyo, Japan), set to represent the vessel lumen filled with contrast material. The LITA graft connected to the $\mathrm{LAD}$, one SVG connected to the PL, and the other to the OM from the aortic root could be viewed clearly (panels A and B). Furthermore, the LAD at the distal portion of the anastomotic site of the LITA could be visualised, suggesting the patency was maintained (upper right panel). Subsequently, conventional bypass graft angiography was performed and good patency of the LITA bypass graft, including the distal portion of the

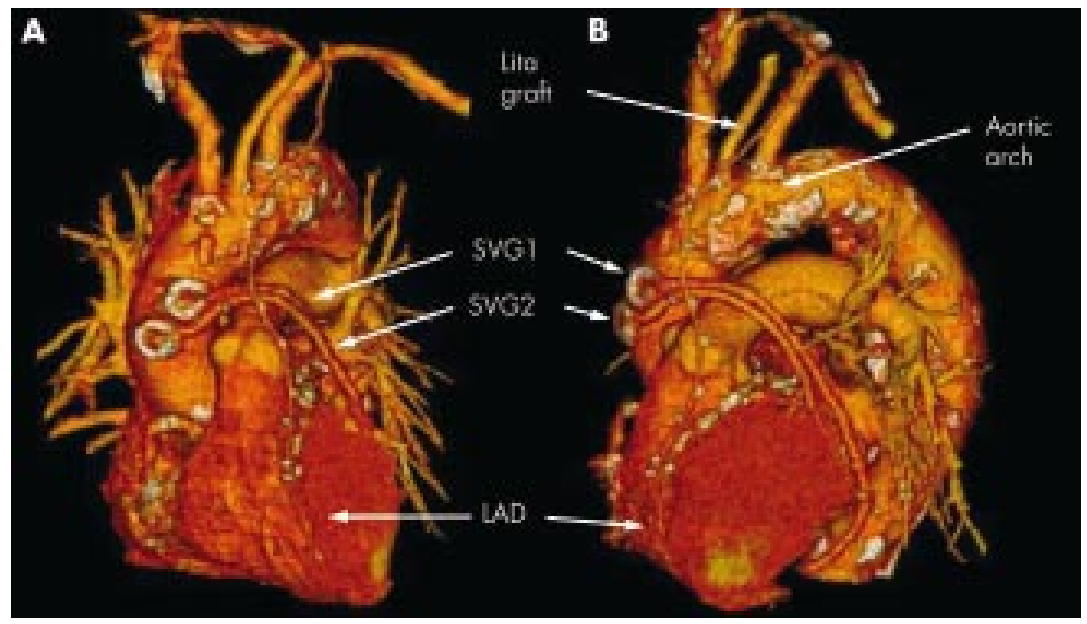

anastomotic sites of the LAD, were confirmed (lower right panel).

N Funabashi

K Teramoto

I Komuro komuro-tky@umin.ac.jp
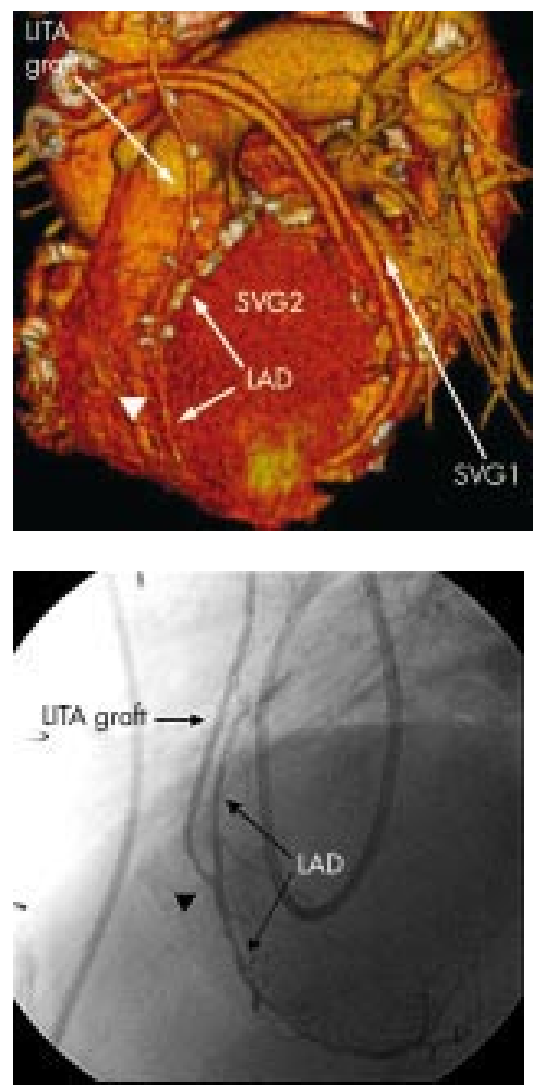